\title{
A Systematic Planning Method for the Electric Vehicles Charging Service Network
}

\author{
Junhai Wang, Leijin Shan, Yongxia Dai, Lingling Ming, and Dayang Yu
}

\begin{abstract}
Electric vehicle (EV) is an important solution for the traffic and environment problems in cities. However, to plan and build the energy supply and service network is always a challenging work all over the world. This paper is based on the planning of a real charge-swap service network for the electric vehicle in Hangzhou city, China. First, the planning factors are analyzed; second, the charging demand forecasting method is proposed based on the future number of EVs; then the service capacity of the single charging station is defined and the planning model of charge-swap service network is proposed which can work for networks including two to three hierarchies.
\end{abstract}

Index Terms-Charge-swap facility, charging network, electric vehicle, planning.

\section{INTRODUCTION}

Charging facility network construction needs continuous development with the extensive application of EVs in fields of public taxis, rental vehicles and official vehicles. The planning and construction of charging facility network must seek the best investment decision to meet the EVs charge-swap demand. The purpose of the study is to determine the reasonable charge-swap network facility planning programs based on the development of EVs and the increase of charge-swap demand. Planning programs should meet the charge-swap demand and be more economic and reasonable. Charging facility network construction is the premise of the development of EV industry.

Ref. [1] analyzed the factors of EV charging network construction, and proposed some rules of EV charging network planning. Ref. [2], the author considered that EV charging facility had a very optimistic prospect, but it asked for concerted effort with all parties to reduce risk. Ref. [3] established EV charging facilities locating and sizing optimal cost model through residents load conditions. This paper will analyze two types of charge-swap service network and put forwards proposals for the planning schemes of different charge-swap service networks.

\section{DOMESTIC AND INTERNATIONAL DEVELOPMENTS OF CHARGING FACILITY}

At present strong countries in traditional vehicle industry in

Manuscript received February 2, 2014; revised June 19, 2014.

Junhai Wang, Leijin Shan, and Yongxia Dai are with Hangzhou Power Supply Company in Zhejiang provincial electric power company, Hangzhou, China (e-mail: wanghdtv@263.net, 1637919087@qq.com, dyx58@163.com)

Lingling Ming and Dayang Yu are with Laboratory for the Synergistic Dispatch of the Decentralized Electric Power Grid, Shandong University, Jinan, China (e-mail: mingling0104@163.com, yudayang@sdu.edu.cn). world such as the U.S., Japan, Germany and Britain, are actively engaged in producing and popularizing electric vehicles. With the development of EV industry, Charging facility network construction constraints the development of EV industry to a large extent. American government accepts EVs as a major component of national strategy, and plans to build 10,000 charging stations in 12 cities before 2015. Japan will build 2,000,000 ordinary charging stations and 5000 fast charging stations before 2020 considering the development of EVs as the core content of low-carbon revolution.

Many cities such as Beijing, Hangzhou, Shanghai, Tianjin, Nanjing, and Shenzhen have been carrying out construction work of charging facility in recent years. At present, 87 standardized charging stations, 5179 battery charger and 7031 AC charging points have been built covering 26 cities in China which has the most charging facilities of any country in the world [4].

\section{Charging Network PlanNing Evaluation FACTOR ANALYSIS}

\section{A. Introduction of Charging Infrastructure}

EV charging mode generally includes DC rapid charging, AC slow charging, and battery swapping [5]. DC fast chargers charge batteries directly in $\mathrm{EV}$, and batteries will be not replaced. Fast charging emerges higher current and voltage than other charging modes and will take 1-3 hours to charge batteries from the $20 \%$ capacity to $100 \%$ capacity. In AC slow charging mode, batteries are charged by a charger in EV. The charging time is about 6-8 hours normally. In battery swapping mode, batteries are replaced for users in charging station. The whole replacement process needs only a few minutes. The batteries replaced will be charged under low power grid load. There are four types of facilities in charging network based on the above three charging modes: charging pile, battery-charging, and battery-swapping station (CSS), centralized battery-charging station (CCS) and battery-swapping station (BSS), as described next.

EVs which load charger use the AC power for electricity by charging piles and the rated power is under $5 \mathrm{KW}$. The disadvantage of charging pile is that the charging takes a long time and the charging time usually is 6-10 hours. Users usually choose charging piles for charging during the stop process because of the characteristic of slow charging process. Many problems such as scattered layout and small scope of the services still exist in charging piles. Generally speaking, charging piles are mainly installed on parking spaces such as underground garage of residential areas, public parking lot and shopping mall. 
CSS swap fully charged batteries for depleted ones for EVs and eliminate much of the waiting time. The time of the process is only few minutes. CSS can charge the depleted batteries collectively when the grid load is low and can preserve batteries. There are three types of CSS based on the scale of the size of CSS: 100 charger stations, 200 charger stations and 400 charger stations. If it's 100 charger station, the station is equipped with 100 chargers to charge batteries; 200 charger station, the station is equipped with 200 chargers; 400 charger station, the station is equipped with 400 chargers, and so on. The chargers can charge batteries 5 times every day.

CCS and BSS provide energy services for EVs together. CCS charges depleted batteries centrally and carries fully charged batteries for BSS 4 times every day. Then BSS swap fully charged batteries for depleted ones for EVs. According to the experience of EV market, CCS is usually equipped with 3000 chargers and usually charge batteries 2 times every day. A CCS usually carry batteries for 20-25 BSSs. CCS can carry 80 batteries for BSS one time and BSS is supplemented with 4 carrier vehicles to carry batteries.

\section{B. Technology and Structure of Charging Network}

Charging facility network has the mode of battery swapping as the threads of technology. The paper presents the EV charging facility network planning aimed at vehicles which get energy by battery replacement [6].

There are tow types of charge-swap service network in the paper: the two-tier structure service network and the three-tier structure service network. The two-tier structure service network is composed of charging pile and CSS and the three-tier structure service network is composed of charging pile, CSS, CCS and BSS. The three-tier structure service network is developed from the two-tier structure service network.

\section{CHARGING DEMAND FORECASTING}

The paper makes the charging facility network planning program mainly aimed at the types of EV: taxi, official vehicle and private car. According to the empirical data, the present rule of EV operation is represented below.

TABLE I: THE PRESENT RULE OF EV OPERATION

\begin{tabular}{cccc}
\hline \multicolumn{4}{c}{ TABLE I: THE PRESENT RULE OF EV OPERATION } \\
\hline Vehicle types & Taxi & $\begin{array}{c}\text { Official } \\
\text { vehicle }\end{array}$ & Private car \\
\hline $\begin{array}{c}\text { Daily average driving } \\
\text { mileage[km] }\end{array}$ & 400 & 50 & 40 \\
$\begin{array}{c}\text { Single driving range }[\mathrm{km}] \\
\text { Daily charge-swap times }\end{array}$ & 100 & 100 & 100 \\
\hline \hline
\end{tabular}

\section{A. EVs Ownership Forecasting}

The increase of car ownership is closely related to population, per capita income level, the society and economic development and transportation. The growth rate is from low to high at first and then tends to rise slightly. The development of the car in many countries shows that the economy is the major factor in pushing the development of the car. The paper adopts the elastic method to predict the increase of car. The key issue for using the elastic method in the increase of car is the determination of elasticity coefficient. The elasticity coefficient is the ratio of the car's growth rate to the growth rate of GDP per person and is the macro criterion for reflecting the relationship between the development of the car and the national economy development.

The elastic method can predict the changes and development of one factor on the base of the development of the other factor. The paper predicts the growth of the car on the basis of present data of GDP per person [7].

$$
R=R_{G D P} \times \gamma
$$

where $R$ represents the growth rate of car ownership; $R_{G D P}$ denotes the GDP per person growth and $\gamma$ represents the elastic coefficient.

\section{B. Daily Charging Demand of EVs Calculation}

The paper calculates the daily charging demand of EVs according to the forecasts of EVs and the primary operative law of every kind of EV. The charging demand of EVs refers to the number of EVs which need replaced batteries or charge batteries every day. The daily charging demand of EVs is described below.

$$
T=\sum_{i=1}^{n_{c l k}} C_{S} \times C_{H} .
$$

where $T$ represents the daily charging demand of EVs; $C_{S}$ denotes the number of one type of EVs; $C_{H}$ represents the daily charging times and $n_{c l x}$ denotes the number of the kinds of EVs.

At present, most of EVs adopt the charging mode of battery swapping and only a small number of official vehicle and private car charge batteries by charging piles. In this paper, the charging demand of charging piles accounts for twenty percent of the aggregate demand and the daily demand of battery replacement of EVs is eighty percent.

\section{EV Charging FACILITY NETWORK PlanNING}

The layout and construction of charging facility network aimed at sustainable development and based on ensuring supply should ensure that EVs can get energy supply in time. Set of rational layout, proper scale and satisfied requirements as a whole should formed a complete integrated system [8].

\section{A. Single EV Charging Station Actual Service Capability Analysis}

The paper adopts vehicle number to measure the daily service capability of every charge-swap facility. At present, the charging facility is inefficient due to the uneven distribution of charging facilities. The paper cites a single station utilization coefficient to reduce the theoretical value of service capability of every charge-swap facility according to the reduction factor of traffic capacity. The actual service capacity of every facility is described below.

$$
F_{H}=B \times C \div 4 \times \alpha .
$$


where $F_{H}$ represents actual service ability of single station; $B$ denotes the number of battery in a station or the number of battery that CCS carries for BSS one time; $C$ represents the charging times of batteries every day in CSS or the times of carrying batteries from CCS to BSS; 4 denotes 4 batteries loaded in a $\mathrm{EV}$ and $\alpha$ denotes single station utilization coefficient.

According to the present operational status of charging facility network, the utilization of charging pile is very low. A charging pile theoretically can provide $3 \mathrm{EVs}$ with service every day because it usually takes 8 hours to charge an EV. The paper cites a charging pile utilization coefficient to reduce the theoretical value of service capability like the single station utilization coefficient.

$$
F_{C}=3 \times \beta \text {. }
$$

where $F_{C}$ represents the actual daily service capacity of a charging pile and $\beta$ denotes the charging pile utilization coefficient.

According to the present operational status of charging facility network, the value of single station utilization coefficient is 0.8 and the value of single charging pile utilization factor is 0.5 in the paper.

TABLE II: Single Charge-Swap FACILITy SERVICE ABILITY

\begin{tabular}{ccc}
\hline \hline & Facility & $\begin{array}{c}\text { Serviced vehicle number of a } \\
\text { single station }\end{array}$ \\
\hline \multirow{3}{*}{ CSS } & 100 chargers station & 100 \\
& 200 chargers station & 200 \\
BSS & 400 chargers station & 400 \\
CCS & 80 batteries station & 64 \\
& 3000 chargers station & 1200 \\
& Charging pile & 1.5 \\
\hline \hline
\end{tabular}

\section{B. Planning Scheme}

Different types of CSS provide different service capability in the service system of the two-tier structure service network. If it's 100 chargers station, all 100 chargers stations can meet 25 percent of the aggregate demand of battery swapping; 200 chargers station, all 200 chargers stations can meet 50 percent of the aggregate demand of battery swapping; 400 chargers station, all 400 chargers stations can meet 25 percent of the aggregate demand of battery swapping. In the three-tier structure service network, CSS and BSS provide battery-swapping services for EVs together and all the percentage of service of the two types of facilities are fifty percent. The design of the different types of CSS follows the pattern of the two-tier structure service network. The charging facility service network faces the problem of shortage of services supply because of the unreasonable layout of charging facilities from the whole service network point. The paper cites the full-network service utilization coefficient to reduce the full-network service capability.

$$
\begin{aligned}
& N_{H}=\sum_{\mathrm{i}=0}^{n} F_{H} \times \lambda . \\
& N_{C}=\sum_{i=1}^{k} F_{C} \times \delta
\end{aligned}
$$

where $N_{H}$ represents the actual full-network service capacity of battery replacement; $F_{H}$ represents actual service ability of single station; $\lambda$ denotes the full-network service utilization coefficient of the battery swapping service; $N_{C}$ represents the actual full-network service capacity of battery-charging; $F_{C}$ represents the actual daily service capacity of a charge pile; $\delta$ denotes the full-network service utilization coefficient of battery-charging service; $n$ denotes the number of CCS and BSS and $k$ denotes the number of charging piles.

The actual serviced vehicle number of the whole network should not be less than the vehicle number that need swap or charge batteries every day.

\section{CASE STUdY}

The paper studies EV charging service network in Hangzhou city and analyzes planning schemes of two types of charge-swap service network. The paper forecasts that the numbers of electric taxis, electric official vehicle and electric private car, are 500,500, and 2000, respectively, according to the elastic method. The demand of daily charge-swap is described below according to (2) and Table I.

\begin{tabular}{ccc}
\multicolumn{2}{c}{ TABLE III: DAILY CHARGE-SwAP DEMAND } \\
\hline \hline $\begin{array}{c}\text { Daily vehicle } \\
\text { number of } \\
\text { battery charging }\end{array}$ & $\begin{array}{c}\text { Daily vehicle number } \\
\text { of battery swapping }\end{array}$ & $\begin{array}{c}\text { Vehicle number of } \\
\text { aggregate demand }\end{array}$ \\
\hline 466 & 1864 & 2330 \\
\hline \hline
\end{tabular}

The full-network service utilization coefficient of the battery swapping service is 0.8 and the full-network service utilization coefficient of battery-charging service is 0.5 in the paper.

\section{A. The Two Types of Charge-Swap Service Network Planning Scheme}

The two-tier structure service network planning scheme and the three-tier structure service network are designed according toTable II and Table III, which are described in Table IV and Table V.

TABLE IV: The Two-TiEr Structure SERvice Network Planning SCHEME

(a)

\begin{tabular}{ccc}
\hline \hline \multicolumn{2}{c}{ Facility } & 100chargers station \\
\hline The number of facility & 3 \\
\hline \hline & & \\
\hline \hline 200chargers station & 400chargers station & Charging pile \\
\hline 4 & 1 & 518 \\
\hline \hline
\end{tabular}

TABLE V: The Three-Tier Structure Service Network Planning

(a)

\begin{tabular}{ccccc}
\hline \multicolumn{4}{c}{ SCHEME } \\
\hline Facility & 100chargers station \\
\hline \hline The number of facility & \multicolumn{2}{c}{2} \\
\hline \hline $\begin{array}{c}\text { 200chargers } \\
\text { station }\end{array}$ & $\begin{array}{c}\text { 400chargers } \\
\text { station }\end{array}$ & CCS & BSS & $\begin{array}{c}\text { Charging } \\
\text { pile }\end{array}$ \\
\hline 3 & 1 & 1 & 20 & 518 \\
\hline \hline
\end{tabular}

\section{CONCLUSION}

To develop EV industry is an important step to carry through low-carbon policy. The construction of the 
charge-swap service network is the prerequisite and foundation of the promotion for EV industry. There are many factors that affect the EV charge-swap service network planning, such as the charge-swap demand, the operation law of EVs, the mode of energy supply, charging time, etc. Two types of charge-swap service network are proposed in the paper by predicting the charge-swap demand of EVs, and it has been used in Hangzhou city.

There are several further work directions from this research. Firstly, the service network planning would be more accurate if more EV customer behavior and habits are taken into account. Secondly, how to select from two types of charge-swap service network in different regions will be discussed in future studies.

\section{ACKNOWLEDGMENT}

The author thanks the Hangzhou Power Supply Company in China for the support on this study and for the further cooperation.

\section{REFERENCES}

[1] F. Xu and G. Yu, "Tentative analysis of layout of electric vehicle charging stations," East of China Electric Power, vol. 10, pp. 1678-1682, 2009.

[2] K. Y. Jiang, B. Wang, and Y. Zhang, "Discussion of parties responsibility at the beginning of national electric vehicle charging market development," Power Demand Side Management, pp. 73-74, July 2010.

[3] L. F. Cou, Z. F Liu, and H. Zhou, "Modeling algorithm of charging station planning for regional electric vehicle," Modern Electric Power, pp. 44-48, April 2010.

[4] L. M. Zhou, "Study of electric vehicles charging station location planning," Dissertation, Shandong University, 2012, pp. 46-47.

[5] C. E. Hatton, S. K. Beella, J. C. Brezet, and Y. C. Wijnia, "Charging stations for urban settings: The design of a product platform for electric vehicle infrastructure in Dutch cities," World Electric Vehicle Journal, vol. 3, pp. 1-13, 2009.

[6] H. S. Wang, Q. Huang, C. H. Zhang, and A. H. Xia, "A novel approach for the layout of electric vehicle charging station," in Proc. IEEE International Conference on Apperceiving Computing and Intelligence Analysis, 2010, pp. 64-70.

[7] R. M. Wang, T. Cheng, and Y. X. Liu, "Prediction of electric car ownership by elasticity coefficient and thousands ownership," Agricultural Equipment and Vehicle Engineering, vol. 6, pp. 40-43, 2011.

[8] C. Wang, J. Yang, N. Liu, and Y. Mao, "Study on siting and sizing of battery-switch station," in Proc. 4th IEEE International Conference on Electric Utility Deregulation and Restructuring and Power Technologies, 2011, pp. 657-662.

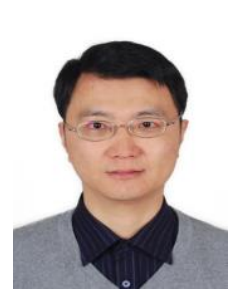

Junhai Wang was born in Zhejiang Province, China, in 1971. He obtained his B.Sc. and M.Sc. degrees from Zhejiang University in motor and control.

He is a senior engineer in Hangzhou Power Supply Company in Zhejiang provincial electric powe company, Hangzhou, China. His research interests include power grid planning and design management and electric vehicle charge-swap facilities operation management.

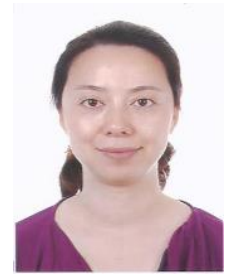

Leijin Shan was born in Zhejiang Province, China, in 1976. She obtained her B.Sc. degree from Zhejiang University.

She is an engineer in Hangzhou Power Supply Company in Zhejiang provincial electric power company. Her research mainly focuses electric vehicle charge-swap facilities operation management.

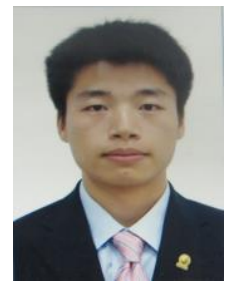

Yongxia Dai was born in Guangxi Province, China, in 1985. He received B.Sc. degree in electrical engineering and its automation from China Jiliang University, Hangzhou, China, in 2007, his M.Sc degree in electrical system and its automation from Shanghai University of Electric Power, Shanghai, China in 2010.

$\mathrm{He}$ is an engineer in Hangzhou Power Supply Company in Zhejiang provincial electric power company. His research mainly focuses electric vehicle charge-swap facilities operation.

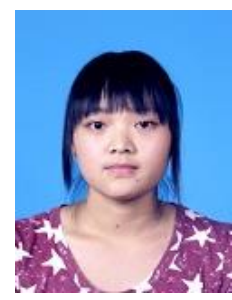

Lingling Ming was born in Taian Province, China, in 1989. She obtained her B.Sc. degree from University of Jinan in 2012. She is currently pursuing the M.Sc degree with School of Electrical Engineering, Shandong University, Jinan, China.

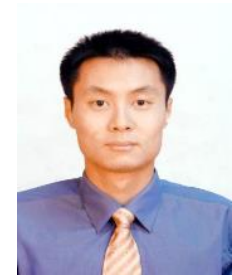

Dayang $\mathbf{Y u}$ is an associate professor in School of Electrical Engineering, Shandong University, Jinan, China. 